\title{
Temporal variability in the biochemical composition of sedimentary organic matter in an intertidal flat of the Galician coast (NW Spain)
}

\section{Variabilité temporelle de la composition biogéochimique de la matière organique du sédiment d'une zone intertidale de la côte de Galice (Espagne NO)}

\author{
Sira Cividanes *, Mónica Incera, Jesús López \\ Departamento de Ecoloxía e Bioloxía Animal, Universidade de Vigo, 36200 Vigo, Spain
}

Received 20 June 2001; received in revised form 31 October 2001; accepted 31 October 2001

\begin{abstract}
Temporal variations and spatial distribution of sedimentary organic matter composition were investigated over a one year period in an intertidal flat of the NW Spain. Sediment samples were collected from 0 to $25 \mathrm{~cm}$ depth, every three months, from January 1997 to January 1998 at three tidal levels (high, medium and low). Changes in the elemental and biochemical composition were assessed to gather information on temporal and spatial fluctuations in quantity and quality of sedimentary organic matter potentially available to benthic deposit-feeder nutrition. Organic matter content was significantly higher at the medium tidal level, while minimum values were found at the high tidal level. The different biochemical classes of organic compounds exhibited different temporal patterns. Carbohydrate and lipid concentrations decreased with sediment depth. The biopolymeric fraction of organic carbon (i.e. the sum of lipid, carbohydrate and protein carbon) was dominated by proteins (61\%), followed by lipids (26\%) and carbohydrates (14\%). Biopolymeric carbon accounted for only a small fraction (37\% on average) of the total organic carbon. Refractory organic carbon (i.e. non biopolymeric) accounted for 50 to $80 \%$ of the total organic carbon and it tended to be buried into deeper sediment layers. The nutritional quality of the sedimentary organic matter, expressed as the biopolymeric carbon to total organic carbon ratio, was higher in January 1997, when also the higher protein to carbohydrate ratio values were observed and related to the presence of newly-produced organic matter. Low biopolymeric carbon to total organic carbon ratio and protein to carbohydrate ratio were recorded during the rest of the year, indicating a low-quality and aged organic matter. Results of the present study revealed an inverse relationship between the overall amount of organic matter and its potential availability to consumers. (C) 2002 Ifremer/CNRS/IRD/Éditions scientifiques et médicales Elsevier SAS. All rights reserved.
\end{abstract}

\section{Résumé}

La distribution spatiale et les variations temporelles de la composition de la matière organique sédimentaire ont été étudiées sur une période d'une année dans une zone intertidale du NO de l'Espagne. Les échantillons ont été récoltés aux profondeurs $0 \mathrm{~cm}$ et $25 \mathrm{~cm}$ chaque trois mois, de janvier 1997 à janvier 1998, à trois moments de la marée (haute, moyenne et basse). Les changements de la composition élémentaire et biochimique ont été suivis pour connaître les fluctuations spatiales et temporelles de la qualité et de la quantité de matière organique disponible pour le benthos. La teneur en matière organique est plus élevée à mi-marée qu'à marée haute. La fraction biopolymérisée du carbone organique (somme du carbone des lipides, des hydrates de carbone et des protéines) est dominée par les protéines $(60,5 \%)$, suivis des lipides $(25,5 \%)$ et des hydrates de carbone $(14,0 \%)$. Ce carbone biopolymérisé représente seulement une faible part $(36,6 \%)$ du carbone organique total. Le carbone organique réfractaire, probablement inutilisable par les animaux, représente entre 50 et $80 \%$ du carbone organique total. La qualité nutritive de la matière organique du sédiment est exprimée par le rapport du carbone biopolymérisé au carbone organique total. Des valeurs élevées de ce rapport sont observées en janvier 1997; ceci indique qu'une large part de la matière organique du sédiment est utilisable par le benthos. Les valeurs élevées du rapport protéines / hydrates de carbone à la même période indiquent la présence d'une matière organique fraîche. À l'inverse, les basses valeurs de ces deux rapports se rencontrent tout le

\footnotetext{
* Corresponding author.

E-mail address: sira@uvigo.es (S. Cividanes).
} 
reste de l'année, ce qui souligne la présence d'une matière organique âgée et de «mauvaise qualité ». Ces données montrent la relation inverse entre la qualité de matière organique et son potentiel pour les dépositivores. (C) 2002 Ifremer/CNRS/IRD/Éditions scientifiques et médicales Elsevier SAS. Tous droits réservés.

Keywords: Sedimentary organic matter; Lipids; Proteins; Carbohydrates; Intertidal sediments

Mots clés: Matière organique sédimentaire; Lipide; Protéine; Hydrate de carbone; Sédiment intertidal

\section{Introduction}

Spatial and temporal changes of sedimentary organic matter $(\mathrm{OM})$ in marine environments affect spatial distribution, metabolism and dynamics of all benthic components, from bacteria to macrofauna Buchanan and Longbottom. 1970: Grant and Hargrave, 1987: Danovaro et al. 1995: Duineveld et al._1997]. As benthic deposit-feeders achieve their food requirement by ingesting sedimentary OM, quantity and composition of sedimentary OM are of primary importance in determining its food availability to consumers Buchanan and Longbottom. 1970: Graf. 1989).

Organic matter in marine environments is composed of labile and refractory compounds whose relative importance might have profound implications for OM diagenesis and turnover Rowe and Deming. 1985; Fabiano et al. 1995). The labile fraction primarily consists of simple sugars and proteins that are rapidly mineralised by bacteria and thus potentially available for higher trophic levels (Eichez, 1991. Danovaro et al. 1993) Conversely, the refractory fraction of $\mathrm{OM}$ is largely composed of complex macromolecules (like humic and fulvic acids and complex polymers), which are degraded slowly, subjected to burial, and thus lost in the short-term for the benthic food webs Eabiano and Danovaro, 1994). Fichez (1991) has proposed the term 'complex organic matter'(COM) to define this residual fraction of the organic carbon, which is not accounted for by lipids, proteins and carbohydrates.

The gross measure of total organic carbon (by CHN analysis) or total organic matter (by ignition loss, Parker 1983 provides only little information on its availability to heterotrophic utilisation Eahiano and Danovare 1994. Newell_and_Field, 1983) Moreover, total organic matter content determined by combustion is generally an overestimate because various inorganic compounds may be oxidised at about $500{ }^{\circ} \mathrm{C}$ (Bretschko and Leichtfried, 1987). In addition, water linked to the silt and clay fractions is not removed by simple drying, but is removed by ignition and accounted for as organic material. As food availability is largely dependent upon OM origin and biochemical composition Tenore and_Hanson, 1980) recent studies have estimated the available fraction of sedimentary organic pools through the determination of the main biochemical classes of organic compounds (i.e. carbohydrates, proteins and lipids), which are assumed to be easier to digest and assimilate Fichez, 1991: Danovaro et al. 1993; Fabiano et bl. 1995: Dell'Anno et al. 2000). Since the relative importance of these biochemical compounds vary in relation to the productivity of the system, the use of biochemical descriptors might provide insights on the trophic potential of marine benthic environments.

The large amount of organic matter reaching the sediments in sheltered intertidal areas are expected to induce a significant benthic response (Josenfson and Conley. 1997. This might explain the high abundance and diversity of the fauna in these environments and their importance as nursery areas for a large number of fish and invertebrate species (Adam, 1990).

Although there are studies on temporal changes in the biochemical composition of sedimentary OM Sargent et al. 1983: Danovaro and Fabiano. 1995: Fabiane et al. 1995: Danovaro, 1996) only little is known about intertidal sediments and comprehensive studies are practically nonexistent. At the same time, the Atlantic intertidal coast of Galicia is subjected to an anthropogenic impact due to seafood exploitation. For these reasons, the knowledge of temporal changes in quantity and quality of sedimentary $\mathrm{OM}$ in intertidal environments is essential to gather information on the system productivity, functioning, benthic efficiency and, in long term, sustainable management.

This study was designed to investigate changes on quantity and quality of sedimentary OM composition as food available for benthic consumers in relation to temporal variations, tidal levels and depths into the sediment.

\section{Materials and methods}

\subsection{Study site}

The studied area is located in the Ria of Arousa, on the Atlantic coast of Galicia (NW Spain, Fig. 1a). Barraña is a sheltered flat characterised by a very low hydrodynamism and influenced by a macrotidal regime (tidal amplitude of 3-4 m). Slight temporal changes were observed in the beach profile throughout the year (Fig. 1b). Deposits of terrigenous material are present along this sandy and muddy intertidal flat ( $350 \mathrm{~m}$ long), which is also characterised by the presence of large amounts of the macroalgae Ulva lactuca. Macrofauna is dominated by the deposit-feeder polychaete Arenicola marina and the bivalves Venerupis decussata, Venerupis pullastra and Cerastoderma edule in the medium and low parts of the flat. Clam and cockle 

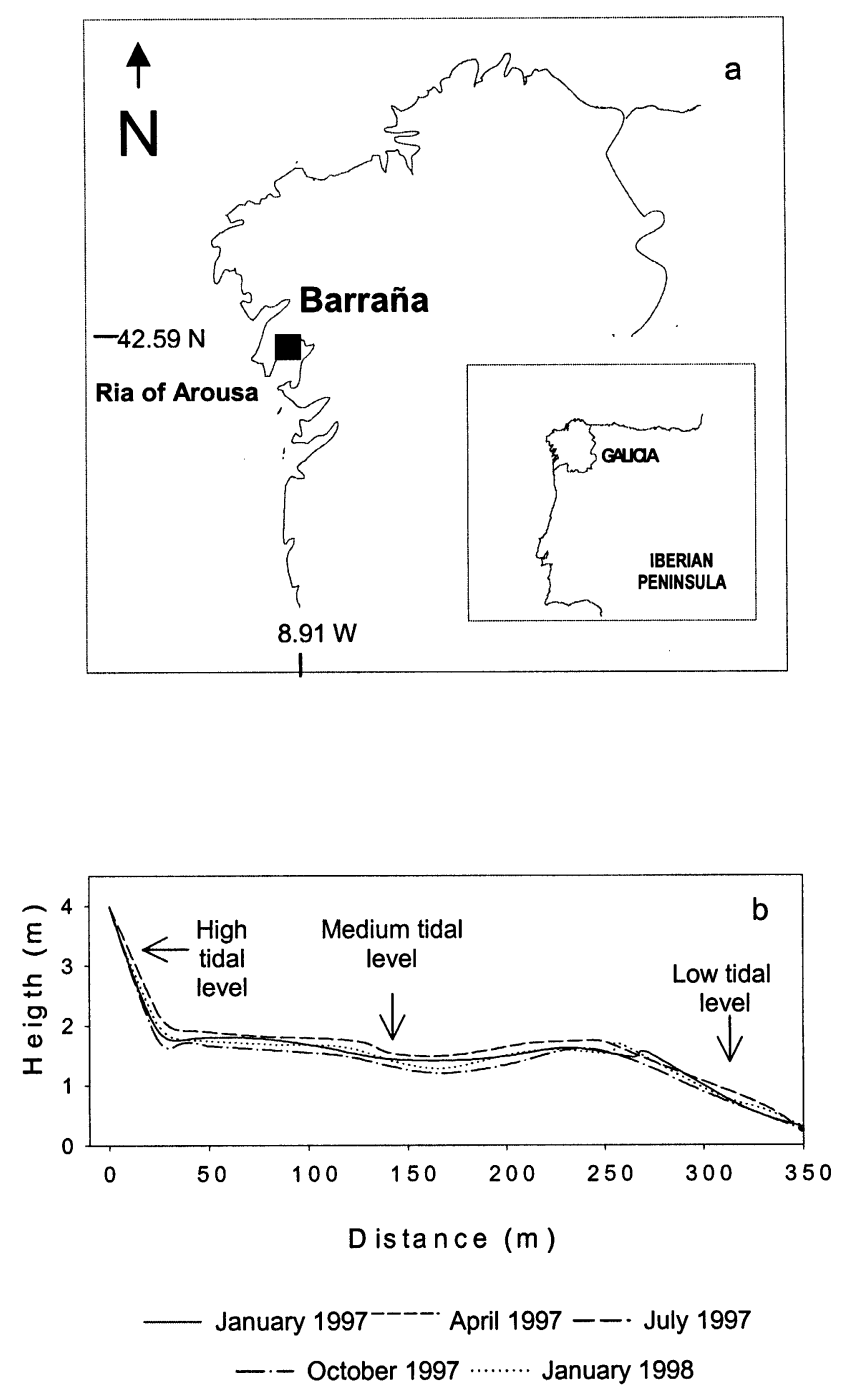

Fig. 1. Location of the intertidal flat (Barraña) on the Galician coast.

collection by hand is a common activity in this highly productive area.

\subsection{Sediment sampling}

Sampling was carried out in January, April, July, October 1997 and January 1998, during spring tide (i.e. tidal range $>3 \mathrm{~m}$ ). Sediment samples were collected in three replicates by hand coring $(15.5 \mathrm{~cm}$ inner diameter core) at three tidal levels (high, medium and low levels, Fig. 1b), after ebbing. The sediment cores were sectioned vertically in the field into 5 depth layers (0-5, 5-10, 10-15, 15-20 and $20-25 \mathrm{~cm}$ depth). Each sediment layer was mixed and subsamples were taken for the analysis of lipids, proteins, carbohydrates, total carbon and nitrogen as well as for carbonates, grain size and water content analysis. All subsamples were frozen at $-30{ }^{\circ} \mathrm{C}$ until further processing, except those for water content analysis.

\subsection{Environmental variables}

Temperature $\left({ }^{\circ} \mathrm{C}\right)$ and redox potential $(\mathrm{mV})$ were measured in situ at $5 \mathrm{~cm}$ intervals down to $25 \mathrm{~cm}$ sediment depth using a probe thermometer and an Eh-electrode connected to a $\mathrm{mV}$ meter, respectively. In addition, the redox potential discontinuity (RPD) was visually estimated as the depth at which the colour of the sediment turns from brown to black (Danovaro, 1996). Sediment shear strength was measured by means of a shear vane meter, Pilcon EDECD, at $5 \mathrm{~cm}$ intervals down to $20 \mathrm{~cm}$ depth. Beach profile was measured each month on the same transect with a Leica NA820 theodolite, according to Emery (1961) Water content in the sediments was estimated as the difference between wet and dry weight $\left(60^{\circ} \mathrm{C}, 72 \mathrm{~h}\right)$ and expressed as percentage. Grain size analysis was carried out by means of a Coulter Counter LS 200. Before analysis, a dried sediment subsample was passed through $2000 \mu \mathrm{m}$ mesh net to retain the coarser fraction; after homogenising, subsamples of about $7 \mathrm{~g}$ were taken and utilised for automatic grain size analysis.

\subsection{Organic carbon and nitrogen}

Total sediment carbon and nitrogen were determined on sediment subsamples (approximately $15-20 \mathrm{mg}$ ) by gaschromatography using a CHNS Fisons Elemental Analyser 1108. Sulfanilamida was used as standard for $\mathrm{C}$ and $\mathrm{N}$. Inorganic carbon was measured using a Bernard Calcimeter as described by Guitian and Carballas (1976) and it was subtracted from total carbon pool to obtain the organic carbon content (Fichez et al._1993.

\subsection{Biochemical composition of the organic matter}

Macrofaunal organisms (i.e. more than $2 \mathrm{~mm}$ ) were removed from the sediment with forceps under binocular before analysis. All the biochemical and elemental analysis were carried out on samples that had been oven-dried at $60{ }^{\circ} \mathrm{C}$ until constant weight and finely powdered with a pestle (Pulverisette 2, Fritsch). After a previous extraction from the dried sediment with 5\% tricloroacetic acid (TCA), total carbohydrates (CHO) were analysed from the supernatant according to Dubois et al (1956) and expressed as glucose equivalents. We selected the Dubois et al (1956) method because the protocol by Gerchacov and Hatcher 1972 largely utilised in deep sea sediments do not work in intertidal sediments due to the interference with carbonates (Cividanes et al., unpublished data). Total protein (PRT) was determined using the method of Lowryet_al_(1951) as modified by Markwell et_al. (1978). We previously tested the differences between this method and others as Rice (1982) method and no significant differences were found between them $(\mathrm{N}=10, P>0.05$ in all cases). Protein concentrations are given as bovine serum albumin (BSA) equivalents. To further investigate the nature of nitrogen, 
protein concentrations were converted to organic nitrogen (N-PRT) by using the conversion factor 6.25 and expressed as percentages of TN (N-PRT: TN*100). Total lipids (LIP) were extracted from dried sediment samples by direct elution with a 2: $1(\mathrm{v} / \mathrm{v})$ chloroform and methanol solution according to Bligh and Dyer (1959). Lipid analysis was carried out according to Marsh and Weinstein (1966) applied to sediments and expressed as tripalmitine equivalents. About 0.5-2.5 g of sediment were used for each analysis and data were normalised to sediment dry weight. Sediment samples combusted at $500{ }^{\circ} \mathrm{C}$ for $6 \mathrm{~h}$ and processed as describe above were utilised as blanks for all biochemical analysis.

\subsection{Quality of the organic matter}

An evaluation of the nutritional value of the sediment was done assuming carbohydrates, proteins and lipids as the more labile compounds of sedimentary OM. The three main biochemical classes were converted to carbon equivalents assuming a conversion factor of $0.45,0.50$ and 0.70 respectively (Fichez, 1991: Fabiano et al. 1995). The sum of lipid, protein and carbohydrate carbon was reported as the biopolymeric carbon fraction (BPC sensu Mayer, 1989. Eichez. 1991: Fabiano and Danovaro, 1994: Fabiano et al. 1995), assumed as a reliable estimate of the labile fraction of total organic matter, i.e., the fraction which was readily available to deposit-feeders. The residual and uncharacterised fraction of the organic carbon (defined here as complex organic matter, COM; Eichez, 1991) was determined as the difference between total organic carbon (TOC) and BPC. The BPC: TOC ratio expressed as a percentage was used as a food index. Protein to carbohydrate ratio (PRT: CHO) were calculated and assumed as estimate of organic material ageing Eabiano et al. 1997.

\subsection{Statistical analyses}

The changes with month, tidal level and depth in biochemical variables were assessed by a three-way Anova analysis Sokal and Rohlf. 1995) A Spearman rank correlation analysis was performed to examine relationships between sediment environmental variables and biochemical composition of OM. A least-square regression analysis was carried out to relate physical, organic, biochemical and other sedimentary variables. SPSS release 10.0 was used for these statistical analyses (Nie_et_al, 1975)

\section{Results}

\subsection{Environmental variables}

Data on temperature, redox potential, shear strength, water content and mean grain size are reported in Table 1.

Table 1

Sediment environmental variables in Barraña between January 1997 and January 1998. (N= 3). (HL, high tidal level; ML, medium tidal level; LL, low tidal level)

\begin{tabular}{|c|c|c|c|c|c|c|c|c|c|c|c|c|c|c|c|c|}
\hline & \multicolumn{4}{|c|}{ Temperature $\left({ }^{\circ} \mathrm{C}\right)$} & \multicolumn{3}{|c|}{ Redox potential (mV) } & \multicolumn{3}{|c|}{ Shear strength $(\mathrm{kPa})$} & \multicolumn{3}{|c|}{ Water content $(\%)$} & \multicolumn{3}{|c|}{ Mean grain size $(\mu \mathrm{m})$} \\
\hline & $\begin{array}{l}\text { Depth } \\
(\mathrm{cm})\end{array}$ & $\mathrm{HL}$ & $\mathrm{ML}$ & LL & $\mathrm{HL}$ & ML & LL & HL & ML & LL & HL & ML & LL & HL & ML & LL \\
\hline \multirow{5}{*}{ Jan 97} & $0-5$ & 13.3 & 12.1 & 13.2 & 224 & 57 & 40 & 3.3 & 4.5 & 5.1 & 21.4 & 23.0 & 25.1 & 341 & 240 & 205 \\
\hline & $5-10$ & 13.2 & 12.0 & 12.8 & 226 & -66 & -158 & 11.3 & 6.7 & 7.5 & 19.9 & 23.5 & 25.4 & 338 & 265 & 198 \\
\hline & $10-15$ & 13.1 & 11.8 & 12.7 & 231 & -103 & -209 & 17.1 & 7.8 & 9.9 & 18.3 & 24.3 & 24.1 & 345 & 311 & 193 \\
\hline & $15-20$ & 12.9 & 11.7 & 12.5 & 242 & -117 & -226 & 20.7 & 12.9 & 13.4 & 18.3 & 22.8 & 24.0 & 417 & 346 & 197 \\
\hline & $20-25$ & n.d. & n.d. & n.d. & 243 & -117 & -226 & & & & 19.2 & 22.8 & 24.7 & 403 & 378 & 220 \\
\hline \multirow{5}{*}{ Apr 97} & $0-5$ & 19.3 & 16.7 & 14.4 & 177 & -163 & -119 & 3.2 & 4.1 & 4.6 & 20.9 & 22.2 & 23.9 & 318 & 233 & 212 \\
\hline & $5-10$ & 19.9 & 16.5 & 14.3 & 211 & -348 & -415 & 5.3 & 6.2 & 10.9 & 20.0 & 21.8 & 23.2 & 328 & 248 & 202 \\
\hline & $10-15$ & 20.2 & 16.4 & 14.5 & 226 & -347 & -505 & 10.1 & 9.9 & 15.9 & 19.1 & 21.6 & 23.0 & 347 & 285 & 198 \\
\hline & $15-20$ & 19.4 & 16.0 & 14.7 & 263 & -443 & -530 & 14.0 & 13.8 & 22.0 & 17.9 & 21.8 & 22.8 & 359 & 340 & 206 \\
\hline & $20-25$ & 18.1 & 15.7 & 14.9 & 276 & -478 & -532 & & & & 18.3 & 22.4 & 23.6 & 404 & 408 & 210 \\
\hline \multirow{5}{*}{ Jul 97} & $0-5$ & 18.6 & 15.8 & 17.9 & 186 & -155 & -125 & 2.3 & 3.8 & 3.4 & 19.2 & 22.3 & 24.1 & 368 & 218 & 191 \\
\hline & $5-10$ & 20.1 & 15.5 & 16.8 & 200 & -365 & -394 & 5.9 & 6.5 & 8.7 & 18.6 & 21.4 & 23.4 & 366 & 250 & 188 \\
\hline & $10-15$ & 21.3 & 15.4 & 16.1 & 237 & -330 & -531 & 8.5 & 9.2 & 14.3 & 17.8 & 22.3 & 23.8 & 356 & 260 & 189 \\
\hline & $15-20$ & 21.9 & 15.7 & 15.9 & 249 & -465 & -503 & 12.2 & 12.4 & 18.1 & 16.4 & 21.8 & 23.0 & 398 & 318 & 192 \\
\hline & $20-25$ & 22.2 & 16.0 & 15.9 & 289 & -454 & -559 & & & & 17.7 & 23.5 & 23.3 & 403 & 402 & 222 \\
\hline \multirow{5}{*}{ Oct 97} & $0-5$ & 17.3 & 17.4 & 17.6 & 210 & -175 & -282 & 3.3 & 5.2 & 3.2 & 19.9 & 21.5 & 21.5 & 359 & 239 & 213 \\
\hline & $5-10$ & 17.5 & 17.4 & 17.6 & 205 & -228 & -305 & 5.7 & 8.5 & 9.3 & 19.2 & 20.7 & 21.8 & 335 & 265 & 221 \\
\hline & $10-15$ & 17.5 & 17.5 & 17.8 & 221 & -231 & -313 & 7.3 & 11.0 & 13.5 & 17.2 & 21.4 & 22.3 & 403 & 314 & 220 \\
\hline & $15-20$ & 17.6 & 17.5 & 17.8 & 228 & -261 & -318 & 11.7 & 12.9 & 18.1 & 17.4 & 21.5 & 22.5 & 367 & 379 & 238 \\
\hline & $20-25$ & 17.6 & 17.6 & 17.7 & 225 & -278 & -327 & & & & 18.2 & 22.6 & 22.3 & 414 & 452 & 262 \\
\hline \multirow{5}{*}{ Jan 98} & $0-5$ & 10.9 & 13.0 & 13.5 & 215 & -218 & -176 & 4.9 & 2.6 & 1.8 & 20.9 & 22.8 & 23.7 & 410 & 221 & 214 \\
\hline & $5-10$ & 11.3 & 13.1 & 13.4 & 225 & -405 & -386 & 7.7 & 6.1 & 5.4 & 19.3 & 22.5 & 23.6 & 409 & 242 & 208 \\
\hline & $10-15$ & 11.6 & 13.1 & 13.4 & 220 & -451 & -470 & 8.4 & 7.0 & 7.9 & 18.8 & 21.9 & 23.4 & 395 & 261 & 213 \\
\hline & $15-20$ & 11.7 & 13.1 & 13.5 & 220 & -473 & -512 & 8.9 & 8.3 & 10.2 & 18.9 & 22.1 & 23.5 & 379 & 377 & 207 \\
\hline & $20-25$ & 11.8 & n.d. & 13.6 & 207 & -470 & -530 & & & & 18.1 & 21.5 & 24.1 & 408 & 341 & 209 \\
\hline
\end{tabular}


Table 2

Summary of three-way Anova of temperature $(\mathrm{T})$, redox potential $(\mathrm{Eh})$, shear strength $(\mathrm{SS})$, water content $(\mathrm{WC})$ and mean grain size $(\mathrm{GS})$ data. $\mathrm{N}=225$. Significance levels: $*: \mathrm{p}<0.05, * *: \mathrm{p}<0.01, * * *: \mathrm{p}<0.001, \mathrm{~ns}: \mathrm{p}>0.05$

\begin{tabular}{|c|c|c|c|c|c|c|c|c|c|c|}
\hline \multirow[t]{2}{*}{ Source of variation } & \multicolumn{2}{|c|}{$\mathrm{T}$} & \multicolumn{2}{|c|}{$\mathrm{EH}$} & \multicolumn{2}{|c|}{ SS } & \multicolumn{2}{|c|}{ WC } & \multicolumn{2}{|c|}{ GS } \\
\hline & $\mathrm{F}$ & $\mathrm{p}$ & $\mathrm{F}$ & $\mathrm{p}$ & $\mathrm{F}$ & $\mathrm{p}$ & $\mathrm{F}$ & $\mathrm{p}$ & $\mathrm{F}$ & $\mathrm{p}$ \\
\hline Month & 768.4 & $* * *$ & 54.1 & $* * *$ & 40.2 & $* * *$ & 13.9 & $* * *$ & 6.3 & $* * *$ \\
\hline Tidal level & 193.7 & $* * *$ & 1484.6 & $* * *$ & 52.5 & $* * *$ & 322.2 & $* * *$ & 672.5 & $* * *$ \\
\hline Depth & 0.4 & ns & 41.9 & $* * *$ & 431.7 & $* * *$ & 6.5 & $* * *$ & 60.7 & $* * *$ \\
\hline Month*Tidal level & 122.8 & $* * *$ & 14.0 & $* * *$ & 35.3 & $* * *$ & 2.3 & $*$ & 4.3 & $* * *$ \\
\hline Month*Depth & 2.3 & $* *$ & 2.1 & $*$ & 7.3 & $* * *$ & 0.5 & ns & 1.5 & ns \\
\hline Tidal level*Depth & 4.7 & $* * *$ & 18.2 & $* * *$ & 7.1 & $* * *$ & 3.8 & $* * *$ & 21.0 & $* * *$ \\
\hline Month*Tidal level*Depth & 4.5 & $* * *$ & 1.3 & $\mathrm{~ns}$ & 4.6 & $* * *$ & 0.5 & ns & 1.2 & ns \\
\hline
\end{tabular}

Table 2

Summary of three-way Anova of protein (PRT), carbohydrate (CHO), lipid (LIP), carbon of the biopolymeric fraction (BPC), total organic carbon (TOC), complex organic matter (COM), BPC:TOC ratio, carbon to nitrogen ratio $(\mathrm{C}: \mathrm{N})$, total nitrogen (TN), contribution of the protein-nitrogen to TN (N-PRT:TN) and protein to carbohydrate ratio (PRT:CHO) data. $\mathrm{N}=225$. Significance levels: $*: \mathrm{p}<0.05, * *: \mathrm{p}<0.01, * * *: \mathrm{p}<0.001, \mathrm{~ns}: \mathrm{p}>0.05$

\begin{tabular}{|c|c|c|c|c|c|c|c|c|c|c|c|c|c|c|c|c|c|c|c|c|c|c|}
\hline \multirow[t]{2}{*}{ Source of variation } & \multicolumn{2}{|c|}{ PRT } & \multicolumn{2}{|c|}{$\mathrm{CHO}$} & \multicolumn{2}{|c|}{ LIP } & \multicolumn{2}{|c|}{$\mathrm{BPC}$} & \multicolumn{2}{|c|}{ PRT: CHO } & \multicolumn{2}{|c|}{ TOC } & \multicolumn{2}{|c|}{ COM } & \multicolumn{2}{|c|}{ BPC: TOC } & \multicolumn{2}{|c|}{$\mathrm{C}: \mathrm{N}$} & \multicolumn{2}{|c|}{ TN } & \multicolumn{2}{|c|}{ N-PRT: TN } \\
\hline & $\mathrm{F}$ & $\mathrm{p}$ & $\mathrm{F}$ & $\mathrm{p}$ & $\mathrm{F}$ & $\mathrm{p}$ & $\mathrm{F}$ & $\mathrm{p}$ & $\mathrm{F}$ & $\mathrm{p}$ & $\mathrm{F}$ & $\mathrm{p}$ & $\mathrm{F}$ & $\mathrm{p}$ & $\mathrm{F}$ & $\mathrm{p}$ & $\mathrm{F}$ & $\mathrm{p}$ & $\mathrm{F}$ & $\mathrm{p}$ & $\mathrm{F}$ & $\mathrm{p}$ \\
\hline Month & 45.7 & $* * *$ & 115.5 & $* * *$ & 8.6 & $* * *$ & 11.8 & $* * *$ & 255.1 & $* * *$ & 6.4 & $* * *$ & 11.5 & $* * *$ & 5.1 & $* * *$ & 6.0 & $* * *$ & 19.6 & $* * *$ & 117.2 & $* * *$ \\
\hline Tidal level & 415.5 & $* * *$ & 719.8 & $* * *$ & 208.1 & $* * *$ & 458.9 & $* * *$ & 36.8 & $* * *$ & 413.4 & $* * *$ & 224.9 & $* * *$ & 3.1 & $*$ & 66.5 & $* * *$ & 330.1 & $* * *$ & 56.7 & $* * *$ \\
\hline Depth & 2.1 & ns & 12.2 & $* * *$ & 16.8 & $* * *$ & 10.5 & $* * *$ & 3.7 & $* *$ & 3.4 & $* *$ & 7.9 & $* * *$ & 1.6 & ns & 8.1 & $* * *$ & 13.1 & $* * *$ & 2.3 & ns \\
\hline Month*Tidal level & 24.4 & $* * *$ & 31.2 & $* * *$ & 13.3 & $* * *$ & 9.5 & $* * *$ & 26.4 & $* * *$ & 10.1 & $* * *$ & 10.8 & $* * *$ & 4.0 & $* * *$ & 3.1 & $* *$ & 5.3 & $* * *$ & 15.3 & $* * *$ \\
\hline Month*Depth & 1.8 & $*$ & 1.7 & ns & 1.2 & ns & 1.7 & ns & 2.6 & $* * *$ & 0.9 & ns & 1.5 & ns & 1.03 & ns & 0.6 & ns & 1.3 & ns & 2.5 & $* *$ \\
\hline Tidal level*Depth & 1.2 & ns & 1.1 & ns & 3.6 & $* * *$ & 2.6 & $* *$ & 2.2 & $*$ & 5.8 & $* * *$ & 5.9 & $* * *$ & 1.3 & ns & 5.5 & $* * *$ & 2.9 & $* *$ & 2.6 & $* *$ \\
\hline $\begin{array}{l}\text { Month*Tidal } \\
\text { level*Depth }\end{array}$ & 1.8 & $* *$ & 1.2 & ns & 2.5 & $* * *$ & 2.2 & $* * *$ & 2.2 & $* * *$ & 1.3 & ns & 1.9 & $* *$ & 1.3 & $\mathrm{~ns}$ & 0.9 & $\mathrm{~ns}$ & 1.3 & $\mathrm{~ns}$ & 3.1 & $* * *$ \\
\hline
\end{tabular}

Temperature showed a well-defined seasonal pattern ranging from 10.9 (January 1998, high tidal level = HL, 0-5 cm depth) to $22.2^{\circ} \mathrm{C}$ (July 1997 , HL, 20-25 cm). Redox potential varied strongly with tidal level, from positive values at the high tidal level (maximum of $276 \mathrm{mV}$, April 1997, $20-25 \mathrm{~cm}$ ) to negative values at the medium and low tidal levels (minimum of $-559 \mathrm{mV}$, July 1997, low tidal level $=\mathrm{LL}, 20-25 \mathrm{~cm}$ ). RPD was observed at $5 \mathrm{~cm}$ depth at the medium and low tidal levels. Shear strength ranged from 1.8 (January 1998, LL, 0-5 cm) to $22 \mathrm{kPa}$ (April 1997, LL, 15-20 cm). Water content ranged between 16.4 (July 1997, HL, 15-20 cm) and 25.4\% (January 1997, LL, 5-10 cm). Mean grain size varied between 188 (July 1997, LL, 5-10 cm) and $410 \mu \mathrm{m}$ (January 1998, HL, 0-5 cm). Threeway Anova analysis showed that all these variables varied significantly with month, tidal level and depth $(\mathrm{N}=225$, $P<0.001$, except for temperature with depth, $P>0.05$, Table 2). Temperature and redox potential decreased with sediment depth while shear strength and mean grain size increased with sediment depth (Table 1).

\subsection{Elemental composition of sedimentary organic matter}

Total nitrogen $(\mathrm{TN})$ ranged from $50 \mu \mathrm{g} \mathrm{g}^{-1}$ (April 1997, HL, $10-15 \mathrm{~cm}$ depth) to $803 \mu \mathrm{g} \mathrm{g}^{-1}$ (July 1997, medium tidal level $=\mathrm{ml}, 0-5 \mathrm{~cm}$ ) $($ Table 3 ). N-PRT accounted for $49 \%$ of total nitrogen on annual average and ranged from $39 \mu \mathrm{g} \mathrm{g}^{-1}$ (July 1997, HL, 15-20 cm) to $642 \mu \mathrm{g} \mathrm{g}^{-1}$ (January $1997, \mathrm{ml}, 10-15 \mathrm{~cm})$. Seasonal variations of total organic carbon (TOC) concentrations and carbon to nitrogen ratio
(C: N) values at each tidal level and sediment depth are illustrated in Fig. 2. TOC concentrations showed a significant seasonality at the medium tidal level $(\mathrm{N}=75$, $P<0.001)$ with a maximum in summer $\left(8.3 \mathrm{mg} \mathrm{g}^{-1}, 20\right.$ $25 \mathrm{~cm})$ and a minimum in winter $\left(2.1 \mathrm{mg} \mathrm{g}^{-1}\right.$, January 1997 , $0-5 \mathrm{~cm})$, while at the high and low tidal levels no significant differences among seasons $(\mathrm{N}=75, P>0.05)$ were evidenced. Highest $\mathrm{C}$ : $\mathrm{N}$ ratio values were recorded at the medium tidal level and they ranged between 4.4 (January 1997, 0-5 cm) and 16.2 (April 1997, 20-25 cm).

\subsection{Biochemical composition of sedimentary organic matter}

Carbohydrate, protein and lipid concentrations showed different temporal patterns (Fig. 3). Concentrations of the three biochemical compounds were significantly higher at the medium tidal level $(\mathrm{N}=225, P<0.001)$. The average carbohydrate concentration during the study period was $239 \mu \mathrm{g} \mathrm{g}^{-1}$ sedDW, with maximum values in July $\left(675 \mu \mathrm{g} \mathrm{g}^{-1}, \mathrm{ml}, \quad 5-10 \mathrm{~cm}\right.$ depth) and October $\left(671 \mu \mathrm{g} \mathrm{g}^{-1}, \mathrm{ml}, 0-5 \mathrm{~cm}\right)$ and minimum in January 1997 (32 $\left.\mu \mathrm{g} \mathrm{g}^{-1}, \mathrm{HL}, 15-20 \mathrm{~cm}\right)$. Protein concentrations (annual average $1037 \mu \mathrm{g} \mathrm{g}^{-1}$ ) showed maximum values in January and April 1997 (4016 $\mu \mathrm{g} \mathrm{g}^{-1}, 15-20 \mathrm{~cm}$ and $3443 \mu \mathrm{g} \mathrm{g}^{-1}$, $5-10 \mathrm{~cm}, \mathrm{ml}$, respectively) and lower values during the rest of the year. Lipid concentrations (annual average of $437 \mu \mathrm{g} \mathrm{g}^{-1}$ ) ranged from $50 \mu \mathrm{g} \mathrm{g}^{-1}$ (January 1998, HL, $15-20 \mathrm{~cm}$ ) to $1483 \mu \mathrm{g} \mathrm{g}^{-1}$ (October 1997, ml, 0-5 cm). Carbohydrate and lipid concentrations decreased with sediment 
Table 3

Total nitrogen (TN), protein-nitrogen content (N-PRT) and contribution of the protein-nitrogen to TN (N-PRT:TN) in Barraña. Mean \pm Standard deviation (N=3). (HL, high tidal level; ML, medium tidal level; LL, low tidal level)

\begin{tabular}{|c|c|c|c|c|c|c|c|c|c|c|}
\hline & \multirow[b]{2}{*}{ Depth $(\mathrm{cm})$} & \multicolumn{3}{|c|}{$\mathrm{TN}\left(\mu \mathrm{g} \mathrm{g}^{-1}\right.$ sed.DW) } & \multicolumn{3}{|c|}{ N-PRT ( $\mu \mathrm{g} \mathrm{g}^{-1}$ sed·DW) } & \multicolumn{3}{|c|}{ N-PRT: TN (\%) } \\
\hline & & HL & ML & LL & HL & ML & LL & $\mathrm{HL}$ & ML & LL \\
\hline \multirow{5}{*}{ Jan 97} & $0-5$ & $116.7 \pm 20.8$ & $520.0 \pm 91.7$ & $426.7 \pm 70.2$ & 68.2 & 374.7 & 221.9 & 58.5 & 72.1 & 52.0 \\
\hline & $5-10$ & $235.0 \pm 48.5$ & $270.0 \pm 144.2$ & $316.7 \pm 65.1$ & 77.9 & 603.2 & 195.4 & 33.2 & 223.4 & 61.7 \\
\hline & $10-15$ & $143.3 \pm 49.3$ & $436.7 \pm 311.3$ & $276.7 \pm 23.1$ & 62.1 & 642.6 & 148.0 & 43.3 & 147.2 & 53.5 \\
\hline & $15-20$ & $196.7 \pm 98.7$ & $490.0 \pm 141.8$ & $283.3 \pm 40.4$ & 61.3 & 445.0 & 124.2 & 31.2 & 90.8 & 43.8 \\
\hline & $20-25$ & $170.0 \pm 113.1$ & $465.0 \pm 21.2$ & $255.0 \pm 49.5$ & 67.2 & 509.1 & 136.0 & 39.5 & 109.5 & 53.3 \\
\hline \multirow{5}{*}{ Apr 97} & $0-5$ & $96.7 \pm 15.3$ & $503.3 \pm 32.1$ & $286.7 \pm 20.8$ & 74.4 & 526.5 & 180.3 & 77.0 & 104.6 & 62.9 \\
\hline & $5-10$ & $70.0 \pm 20.0$ & $620.0 \pm 70.0$ & $213.3 \pm 20.8$ & 56.3 & 550.9 & 106.7 & 80.5 & 88.9 & 50.0 \\
\hline & $10-15$ & $50.0 \pm 0.01$ & $473.3 \pm 56.9$ & $210.0 \pm 10.0$ & 52.0 & 472.5 & 112.2 & 103.9 & 99.8 & 53.4 \\
\hline & $15-20$ & $56.7 \pm 15.3$ & $490.0 \pm 91.7$ & $200.0 \pm 10.0$ & 58.8 & 413.3 & 84.5 & 103.7 & 84.3 & 42.2 \\
\hline & $20-25$ & $60.0 \pm 20.0$ & $363.3 \pm 77.7$ & $160.0 \pm 43.6$ & 56.7 & 383.0 & 96.0 & 94.5 & 105.4 & 60.0 \\
\hline \multirow{5}{*}{ Jul 97} & $0-5$ & $266.7 \pm 20.8$ & $803.3 \pm 64.3$ & $443.3 \pm 15.3$ & 67.2 & 221.9 & 131.3 & 25.2 & 27.6 & 29.6 \\
\hline & $5-10$ & $223.3 \pm 15.3$ & $716.7 \pm 98.7$ & $380.0 \pm 43.6$ & 61.2 & 260.5 & 89.3 & 27.4 & 36.4 & 23.5 \\
\hline & $10-15$ & $213.3 \pm 40.4$ & $730.0 \pm 113.6$ & $323.3 \pm 11.5$ & 55.4 & 222.3 & 63.4 & 26.0 & 30.5 & 19.6 \\
\hline & $15-20$ & $230.0 \pm 95.4$ & $556.7 \pm 15.3$ & $296.7 \pm 5.8$ & 39.5 & 200.6 & 61.0 & 17.2 & 36.0 & 20.6 \\
\hline & $20-25$ & $216.7 \pm 15.3$ & $555.0 \pm 63.6$ & $353.3 \pm 35.1$ & 61.6 & 164.2 & 68.0 & 28.4 & 29.6 & 19.2 \\
\hline \multirow{5}{*}{ Oct 97} & $0-5$ & $213.3 \pm 49.3$ & $726.7 \pm 51.3$ & $406.7 \pm 90.7$ & 73.7 & 283.2 & 108.2 & 34.5 & 39.0 & 26.6 \\
\hline & $5-10$ & $166.7 \pm 46.2$ & $696.7 \pm 20.8$ & $283.3 \pm 75.1$ & 79.9 & 263.3 & 50.4 & 47.9 & 37.8 & 17.8 \\
\hline & $10-15$ & $160.0 \pm 65.6$ & $736.7 \pm 189.0$ & $323.3 \pm 20.8$ & 76.4 & 158.3 & 56.9 & 47.7 & 21.5 & 17.6 \\
\hline & $15-20$ & $95.0 \pm 7.1$ & $636.7 \pm 70.2$ & $346.7 \pm 35.1$ & 53.1 & 179.6 & 54.0 & 55.9 & 28.2 & 15.6 \\
\hline & $20-25$ & $136.7 \pm 40.4$ & $540.0 \pm 60.8$ & $400.0 \pm 28.3$ & 79.2 & 210.2 & 42.5 & 58.0 & 38.9 & 10.6 \\
\hline \multirow{5}{*}{ Jan 98} & $0-5$ & $150.0 \pm 10.0$ & $546.7 \pm 136.1$ & $280.0 \pm 17.3$ & 82.7 & 185.6 & 73.2 & 55.1 & 33.9 & 26.1 \\
\hline & $5-10$ & $183.3 \pm 32.1$ & $430.0 \pm 60.8$ & $323.3 \pm 46.2$ & 78.8 & 154.9 & 88.2 & 43.0 & 36.0 & 27.3 \\
\hline & $10-15$ & $113.3 \pm 25.2$ & $530.0 \pm 124.9$ & $296.7 \pm 35.1$ & 60.8 & 191.2 & 99.7 & 53.7 & 36.1 & 33.6 \\
\hline & $15-20$ & $255.0 \pm 63.6$ & $496.7 \pm 95.0$ & $256.7 \pm 20.8$ & 65.7 & 328.2 & 72.0 & 25.8 & 66.1 & 28.1 \\
\hline & $20-25$ & $130.0 \pm 14.1$ & $456.7 \pm 145.7$ & $273.3 \pm 15.3$ & 56.1 & 329.8 & 81.0 & 43.2 & 72.2 & 29.6 \\
\hline
\end{tabular}

depth, while protein content did not show a clear trend. BPC concentrations showed a temporal trend similar to proteins and ranged from $229 \mu \mathrm{g} \mathrm{g}^{-1}$ (April 1997, HL, 10-15 cm) to $2961 \mu \mathrm{g} \mathrm{g}^{-1}$ (April 1997, ml, 5-10 cm) (Fig. 3). PRT: CHO ratio ranged from 1.3 (October $1997, \mathrm{LL}, 5-10 \mathrm{~cm}$ ) to 32.3 (January 1997, ml, 15-20 cm) (Fig. 3). Three-way Anova results showed that all the biochemical variables displayed significant differences with seasons and tidal levels $(\mathrm{N}=225, P<0.001$ for each, Table 2). Carbohydrate and lipid concentrations also showed significant differences with sediment depth $(P<0.001)$. Moreover, there was a significant interaction between month and tidal level for each variable studied $(P<0.001$, Table 2$)$.

Temporal variations of BPC: TOC ratio and complex organic matter content (COM) at different tidal levels and depths are showed in Fig. 2. Concentrations of BPC: TOC ratio and $\mathrm{COM}$ were significantly higher at the medium tidal level $(\mathrm{N}=225, P<0.001)$. The BPC: TOC ratio values indicated that BPC on annual average accounted for a small fraction of the total organic carbon (37\%). The BPC: TOC ratio ranged from 14 (July $1997, \mathrm{ml}, 20-25 \mathrm{~cm}$ ) to $94 \%$ (January 1997, ml, 5-10 cm). COM ranged between $7.1 \mathrm{mg} \mathrm{g}^{-1}$ (July 1997, ml, 20-25 cm) and $0.07 \mathrm{mg} \mathrm{g}^{-1}$ (April 1997, HL, 10-15 cm), and it represented the largest pool for organic carbon (63\% on average). Three-way Anova analysis showed that COM and BPC: TOC ratio displayed significant differences with months $(\mathrm{N}=225$, $P<0.001$, Table 2$)$ and tidal levels $(P<0.001$ and $P<0.05$, respectively). COM also showed significant differences with sediment depth $(P<0.001$, Table 2$)$.

\subsection{Correlation analysis}

The results of the correlation analysis carried out on the environmental and biological variables investigated are reported in Table 4 . The three biochemical compounds were positively inter-correlated $(P<0.01)$. In addition, proteins, carbohydrates and lipids were also positively correlated with most of the variables or parameters representing the nutritive compounds of the sedimentary organic matter, such as $\mathrm{C}$ : $\mathrm{N}$ ratio, TOC, COM and TN $(P<0.01)$. BPC was significantly and negatively correlated with redox potential $(P<0.01)$. Water content was negatively correlated with grain size, redox potential and temperature $(P<0.01)$.

BPC and TOC showed a significant and positive correlation $\left(\mathrm{R}^{2}=0.72, P<0.001\right.$, Fig. $\left.4 \mathrm{a}\right)$. An inverse relationship was found between $\mathrm{C}$ : $\mathrm{N}$ ratio and BPC: TOC ratio $\left(\mathrm{R}^{2}=0.37, P<0.001\right.$, Fig. $\left.4 \mathrm{~b}\right)$.

\section{Discussion}

\subsection{Spatial and temporal changes in organic matter (OM) biochemical composition}

The biochemical composition of sedimentary OM has recently been used to gather information on the origin, 
TOC

$\left(\mathrm{mg} \mathrm{C} \mathrm{g}^{-1} \operatorname{sed} \cdot \mathrm{DW}\right)$
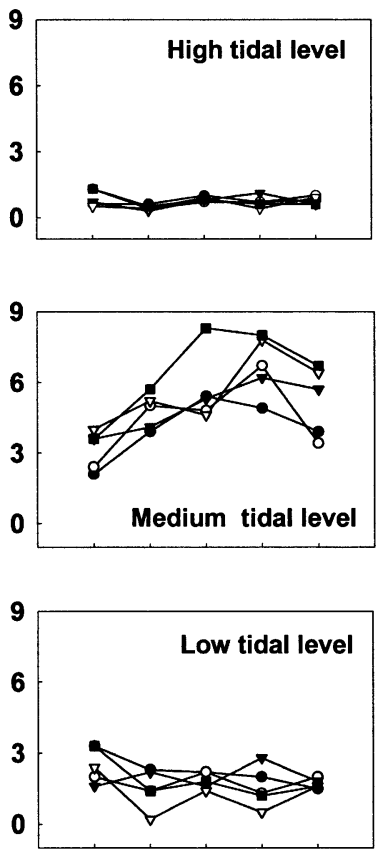

Jan97 Apr Jul Oct Jan98
COM

(mg g ${ }^{-1}$ sed.DW)
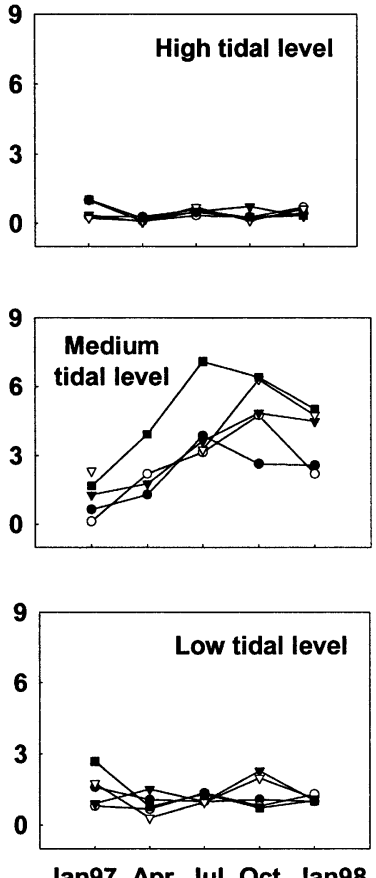

BPC:TOC

(\%)
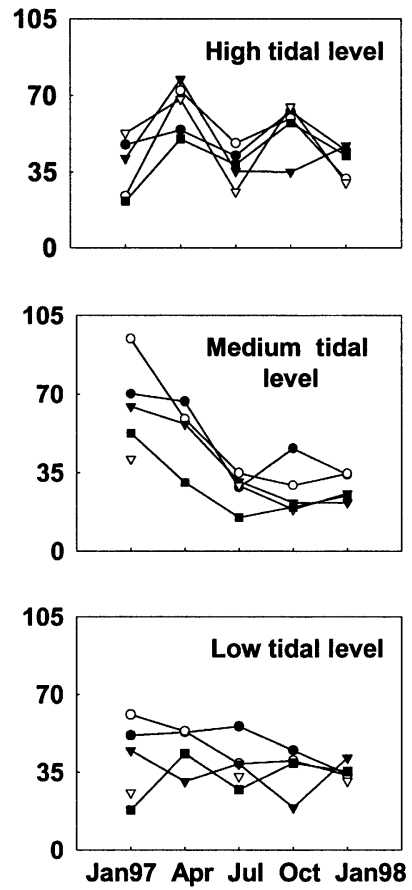

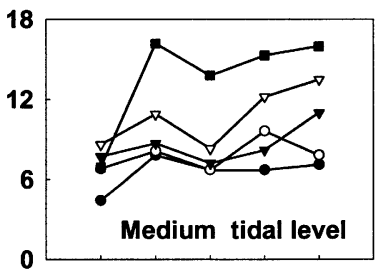

C:N ratio
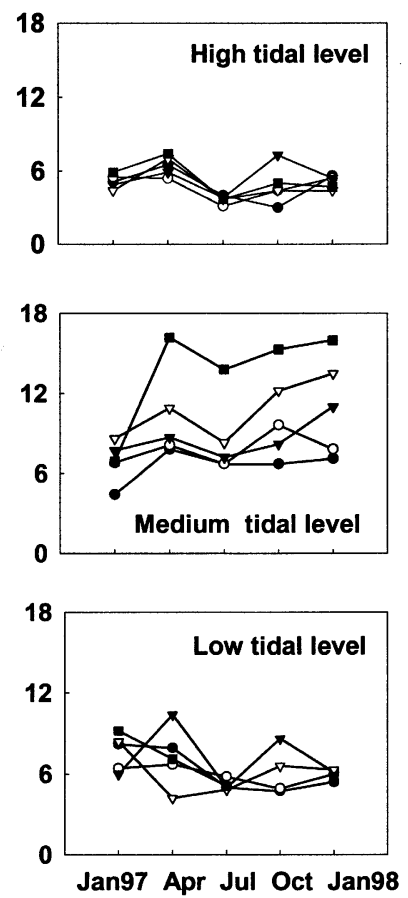

$0-5 \mathrm{~cm}-0-5-10 \mathrm{~cm}$

$10-15 \mathrm{~cm}$

$\rightarrow-15-20 \mathrm{~cm}$

20-25 cm depth

Fig. 2. Temporal changes in total organic carbon (TOC), complex organic matter (COM), biopolymeric carbon to total organic carbon ratio (BPC: TOC) and carbon to nitrogen ratio $(\mathrm{C}: \mathrm{N})$ with tidal levels and sediment depths. Standard deviations are not showed to improve the comprehension of the figures.
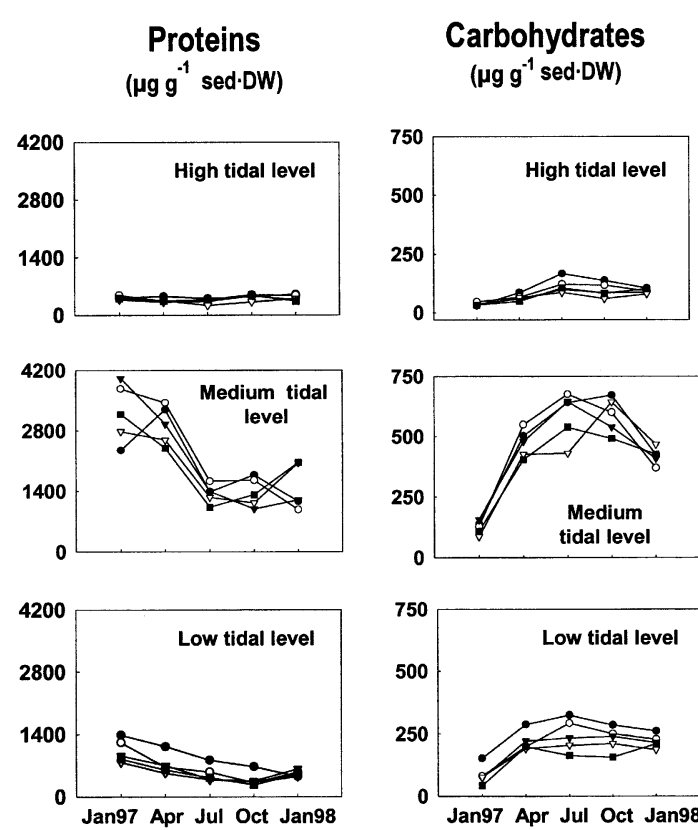
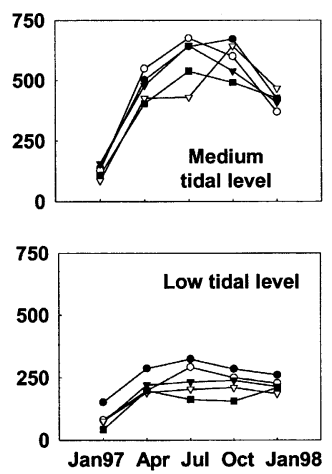

BPC

$\left(\mu g^{-1}\right.$ sed.DW)
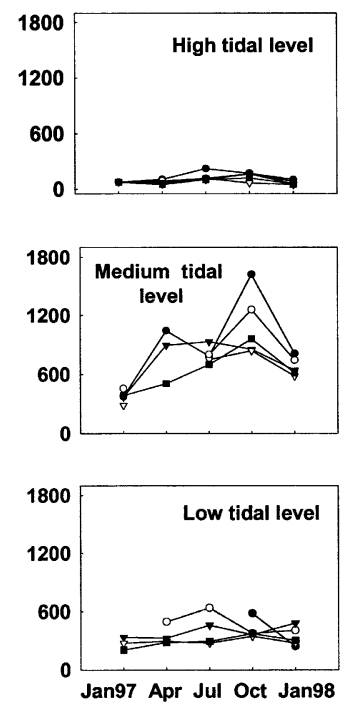
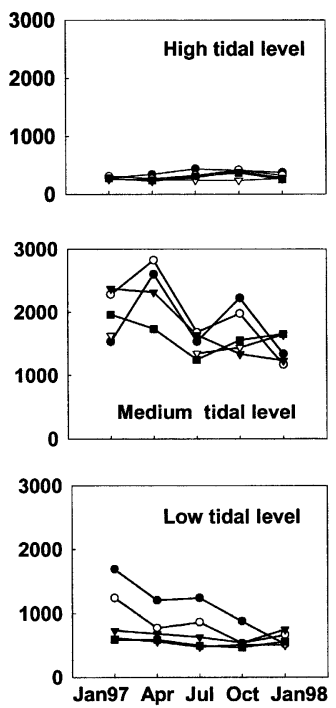

PRT:CHO ratio
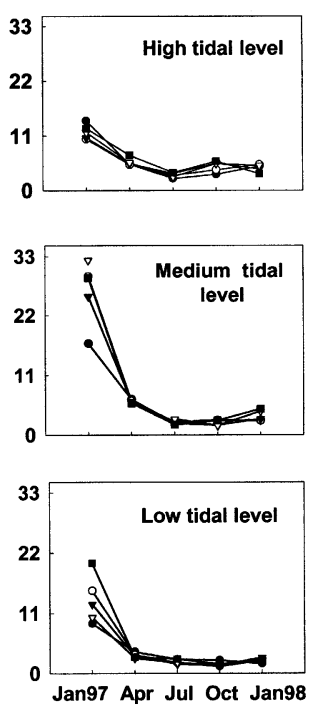

Fig. 3. Temporal variations in the concentrations of carbohydrates, proteins, lipids, biopolymeric carbon (BPC) and protein to carbohydrate ratio (PRT: CHO) with tidal levels and sediment depths. Standard deviations are not showed to improve the comprehension of the figures. 
Table 4

Spearman rank-order correlation analysis between environmental variables and biochemical compounds measured in Barraña: protein (PRT), carbohydrate (CHO), lipid (LIP), protein to carbohydrate ratio (PRT:CHO), carbon of the biopolymeric fraction (BPC), BPC:TOC ratio, carbon to nitrogen ratio (C:N), total organic carbon (TOC), complex organic matter (COM), total nitrogen (TN), water content (WC), mean grain size (GS), redox potential (EH), temperature $(\mathrm{T})$ and shear strength $(\mathrm{SS}) . \mathrm{N}=225$. Significance levels: $*: \mathrm{p}<0.05, * *: \mathrm{p}<0.01$

\begin{tabular}{|c|c|c|c|c|c|c|c|c|c|c|c|c|c|c|}
\hline & PRT & $\mathrm{CHO}$ & LIP & PRT:CHO & $\mathrm{BPC}$ & BPC:TOC & $\mathrm{C}: \mathrm{N}$ & TOC & $\mathrm{COM}$ & $\mathrm{TN}$ & WC & GS & $\mathrm{EH}$ & $\mathrm{T}$ \\
\hline $\mathrm{CHO}$ & $0.557 * *$ & 1.000 & & & & & & & & & & & & \\
\hline LIP & $0.735 * *$ & $0.845^{* *}$ & 1.000 & & & & & & & & & & & \\
\hline PRT:CHO & $0.254 *$ & $-0.602 * *$ & $-0.327 * *$ & $=1.000$ & & & & & & & & & & \\
\hline $\mathrm{BPC}$ & $0.919 * *$ & $0.746 * *$ & $0.899 * *$ & $=-0.054$ & 1.000 & & & & & & & & & \\
\hline BPC:TOC & -0.063 & $-0.297^{*}$ & -0.141 & $0.381 * *$ & -0.095 & 1.000 & & & & & & & & \\
\hline $\mathrm{C}: \mathrm{N}$ & $0.649 * *$ & $0.477 * *$ & $0.561 * *$ & $=0.066$ & $0.641^{* *} *$ & $-0.357 * *$ & 1.000 & & & & & & & \\
\hline TOC & $0.817 * *$ & $0.760 * *$ & $0.831 * *$ & -0.151 & $0.883 * *$ & $-0.492 * *$ & $0.754 * *$ & 1.000 & & & & & & \\
\hline $\mathrm{COM}$ & $0.627 * *$ & $0.734 * *$ & $0.742 * *$ & $-0.329 * *$ & $0.737 * *$ & $-0.632 * *$ & $0.737 * *$ & $0.907 * *$ & 1.000 & & & & & \\
\hline $\mathrm{TN}$ & $0.741 * *$ & $0.792 * *$ & $0.846^{* *}$ & $-0.270^{*}$ & $0.861^{* *} *$ & $-0.336^{* *}$ & $0.537 * *$ & $0.894 * *$ & $0.832 * *$ & 1.000 & & & & \\
\hline WC & $0.467 * *$ & $0.316^{* * *}$ & $0.537 * *$ & -0.114 & $0.580 * *$ & -0.081 & $0.368 * *$ & $0.470 * *$ & $0.428 * *$ & $0.433 * *$ & 1.000 & & & \\
\hline GS & -0.183 & $-0.293^{*}$ & $-0.455^{* *}$ & $=0.300 * *$ & $-0.357 * *$ & -0.031 & -0.073 & -0.212 & -0.223 & $-0.258 *$ & $-0.750 * *$ & 1.000 & & \\
\hline $\mathrm{EH}$ & $-0.383 * *$ & $-0.672 * *$ & $-0.605 * *$ & $=0.491 * *$ & $-0.566^{* * *}$ & $0.360 * *$ & $0.447 * *$ & $-0.554 * *$ & $-0.610 * *$ & $-0.535 * *$ & $-0.648 * *$ & $0.582 * *$ & 1.000 & \\
\hline $\mathrm{T}$ & $-0.392 * *$ & 0.153 & 0.018 & $-0.421 * *$ & -0.229 & 0.132 & -0.165 & -0.207 & -0.150 & -0.123 & $-0.371 * *$ & 0.074 & 0.098 & 1.000 \\
\hline SS & -0.247 & -0.170 & -0.203 & -0.020 & -0.216 & $-0.260^{*}$ & 0.139 & -0.095 & 0.048 & -0.153 & -0.066 & 0.011 & -0.196 & 0.006 \\
\hline
\end{tabular}

quality and food availability of organic matter Danovaroet al. 1993: Fabiano et al. 1995:-Danovare, 1996. The high concentrations of sedimentary proteins, carbohydrates and lipids recorded in the study area (see Table 5 for comparisons) are probably related to the morphodynamic, hydrological and physico-chemical characteristics of this sheltered intertidal flat. Low hydrodynamism favours accumulation of OM mainly due to settlement of organic
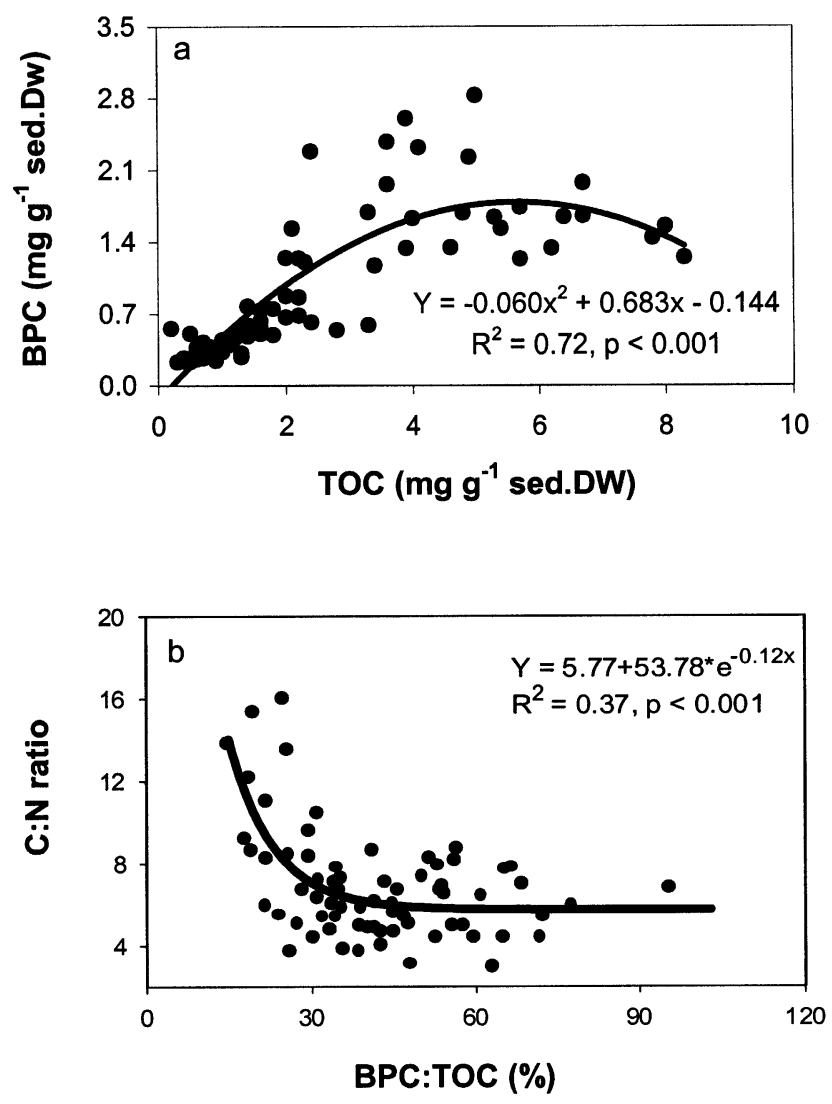

Fig. 4. Relationship between: (a) total organic carbon (TOC) and biopolymeric carbon (BPC); (b) BPC: TOC ratio and C: N ratio. rich fine sediments Nordstrom,1992) In addition, the low energy of the surge allows the formation of fine and stable sediments that permits the settlement of an abundant fauna.

All sedimentary labile organic compounds (i.e. carbohydrates, proteins and lipids) were found to vary strongly between sampling periods (even at greater depths), suggesting that intertidal flats are systems characterised by temporal fluctuations. In Barraña, the temporal changes were more evident over the medium tidal level than in the high or low tidal levels. The high concentrations of biochemical compounds at the medium tidal level can be related to the presence of large amounts of organic matter: macroalgal coverage (Ulva lactuca), deposits of terrigenous and other land-derived material (vascular-plants and wood debris) and polychaete faeces. This was evident from direct field observations during sampling. Our discussion will therefore be focused on the medium tidal level. Lowest concentrations of lipids, proteins and carbohydrates were found at the high tidal level, where OM deposits were not observed. An alternative explanation for such differences among tidal levels could be related to the influence of the presence of clams and cockles, more abundant at the medium tidal level (Cividanes et al., unpublished data).

Temporal variations of carbohydrates and lipids showed completely opposite trends to those of proteins (Fig. 3). The protein content was maximum at January 1997 and decreased until October 1997, while total carbohydrate and lipid concentrations were maximum in October 1997 and minimum in January 1997. These temporal differences between the various biochemical compounds suggest marked changes occurred in composition and/or origin of the OM inputs during the sampling period. These differences were not directly related to temperature, grain size or shear strength, but they were closely correlated with redox potential and water content $(P<0.01$ for both). Carbohy drate maxima at the medium tidal level in July and October coincided with the periods of higher macroalgae coverage 
Table 5

Comparison of carbohydrate, protein and lipid concentrations from different flats and marine systems

\begin{tabular}{|c|c|c|c|c|c|c|}
\hline \multirow[t]{2}{*}{ Location } & & \multirow{2}{*}{$\begin{array}{c}\text { Carbohydrates } \\
\left(\mathrm{mg} \mathrm{g}^{-1} \text { sed·Dw }\right)\end{array}$} & \multirow{2}{*}{$\frac{\text { Proteins }}{\left(\mathrm{mg} \mathrm{g}^{-1} \mathrm{sed} \cdot \mathrm{Dw}\right)}$} & \multirow{2}{*}{$\begin{array}{c}\text { Lipids } \\
\left(\mathrm{mg} \mathrm{g}^{-1} \mathrm{sed} \cdot \mathrm{Dw}\right)\end{array}$} & \multicolumn{2}{|l|}{ Source } \\
\hline & & & & & & \\
\hline \multicolumn{7}{|l|}{ Other flats } \\
\hline Eastern Passage (Halifax, Canada) & sandflat & $0.04-0.08$ & & & Grant et al., & 1986 \\
\hline Cape Henlope (Delaware, USA) & sandflat & & $0.42-0.95$ & & Bock \& Miller, & 1995 \\
\hline Bay of Mont-Saint-Michel (France) & intertidal flat & & & $0.03-0.2$ & Meziane et al., & 1997 \\
\hline Barraña (Galicia, Spain) & intertidal flat & $0.03-0.67$ & $0.4-4.1$ & $0.05-1.48$ & Cividanes et al., & present study \\
\hline Other marine systems & Water depth $(m)$ & & & & & \\
\hline Balsfjorden (Norwey) & & nd & nd & 0.13 & Sargent et al., & 1983 \\
\hline Baltic Sea & 18 & $0.4-4.0$ & $3.8-7.7$ & nd & Meyer-Reil, & 1983 \\
\hline Gulf of Lions & 26 & $0.3-4.3$ & $0.1-2.3$ & nd & Buscail et al., & 1995 \\
\hline Marseille & $6-17$ & $2.4-4.1$ & $1.0-2.6$ & $0.07-0.66$ & Fichez, & 1991 \\
\hline Ligurian Sea (Zoagli) & 10 & $0.13-0.67$ & $0.02-0.07$ & $0.02-0.21$ & Fabiano et al., & 1995 \\
\hline Ligurian Sea (Prelo) & 4 & $0.3-3.6$ & $0.05-1.6$ & $0.10-1.08$ & Danovaro et al., & 1994 \\
\hline Ligurian Sea & 4 & $0.3-5.3$ & $0.02-0.3$ & $0.09-0.63$ & Danovaro \& Fabiano, & 1995 \\
\hline Northern Adriatic Sea & $15-55$ & $0.07-0.42$ & $0.2-4.1$ & $0.07-1.07$ & Manini et al., & 1999 \\
\hline Southern Adriatic Sea & $15-55$ & $0.09-0.64$ & $0.9-3.7$ & $0.14-0.62$ & Manini et al., & 1999 \\
\hline Tyrrhenian Sea & $20-60$ & $0.3-1.9$ & $0.2-1.7$ & $0.001-0.025$ & Fabiano \& Danovaro, & 1994 \\
\hline Ionian and Aegean Seas & $0-2401$ & $1.2-2.5$ & $0.07-0.16$ & $0.05-0.19$ & Danovaro et al., & 1993 \\
\hline
\end{tabular}

(as evident from field observations). The particularly high values of protein concentrations observed in January 1997 and April (but not in January 1998) are difficult to explain. According to Danovaro (1996) high protein concentrations could be attributed to allochthonous inputs. In contrast, protein concentrations were relatively low during the rest of the year.

Carbohydrate and lipid concentrations decreased with sediment depth. This may have been the result of the redox potential discontinuity (RPD) layer being so shallow at $5 \mathrm{~cm}$. However, any such a clear trend was detectable in protein content. This could be due to the accumulation of unutilised proteins that may become absorbed onto organic macromolecules (i.e., COM, Eichez. 1991.

Biochemical composition of sedimentary organic matter seems to be quite different from one marine coastal area to another, but it is usually characterised by small amounts of total lipids and large quantities of proteins, often exceeding carbohydrate concentrations (Meyer-Reil, 1983; Sargent et bl 1983: Fahiano and Danovaro, 1994. Results of the present study are consistent with this pattern. Proteins were the dominant class among labile compounds $(61 \%$ on annual average), followed by lipids (26\%) and carbohydrates $(14 \%)$. Moreover, concentrations of the three components in Barraña were higher than those reported for other intertidal areas Grant et al__1986: Bock and Miller_1995. Meziane et al.,1997, Table 5). Interestingly, biochemical compounds values were similar to those observed in the Adriatic Sea Manini_et_al___1999, Table 5), indicating that the quantity of sedimentary organic matter in intertidal sediments can be comparable with data from other shallow marine basins.

\subsection{Changes in quality of sedimentary organic matter (OM)}

Total organic matter (TOC) concentrations were similar to those reported from other coastal and estuarine areas Danovaro et al. 1994: Danovaro and Fahiano, 1995: Buscailet_al, 1995) but organic carbon composition in Barraña appeared to be highly refractory (63\% on average). During this study, the sedimentary biopolymeric organic carbon (BPC) accounted for $37 \%$ of TOC and decreased from January 1997 (on average 61\%) to October 1997 (on average 25\%). Tenore and Hanson (1980) suggested that about $5-15 \%$ of sedimentary detritus, depending on the environmental characteristics, is generally available at any time for benthic consumers. Data presented here are consistent with these findings. Although the observed temporal changes in the BPC contribution to TOC resulted mainly from the strong temporal variations in total protein concentrations, BPC accounted for only a small fraction of TOC (37\%). Moreover, BPC increased with TOC (Fig. 4a) to a certain level $\left(\sim 3 \mathrm{mgC} \mathrm{g}^{-1}\right)$ above which the fraction potentially utilisable by organisms remained constant or even decreased, due to the presence of high COM concentration.

A similar result is obtained by converting protein content to $\mathrm{N}$ equivalents where proteins account for only $49 \%$ (annual average) of the total nitrogen pool. These values must be viewed with caution since they are obtained by the use of a conversion factor but indicate that a significant fraction of the organic carbon and nitrogen (63 and 51\%, respectively) is geopolymeric (sensu Mayer, 1989) and of less degradable nature (Cough and Mantoura, 1990) that concentrates mainly in the medium level and in deeper 
sediment layers. Thus, although large amounts of detrital organic matter were recorded in Barraña intertidal flat, this detritus was of low nutritional quality. These findings confirm the high amount of COM present in the estuarine sediments. Other authors Saliot. 1984: Ittekkot. 1988 have obtained similar results in other estuarine areas.

A further confirmation of the refractory composition of sedimentary OM was that COM displayed the same temporal and vertical patterns of TOC at all tidal levels (Fig. 2). The high values of COM recorded at the medium tidal level in July and October were coupled to high C: $\mathrm{N}$ ratios (up to 16.2). Such high $C: N$ values suggest the presence of refractory and aged organic matter that has been buried into the sediments Zavattarelli, 1986; Metzler and Smock, 1990. These data also support the hypothesis that COM displays a conservative behaviour in estuaries Utrekkot 1988: Spitzy and Ittekkot. 1991. Decomposition and burial of terrigenous material (C: N > 10; Hargrave et al. 1995) might have also contributed to such concentrations.

The ratio of BPC to TOC has been considered to be a possible indicator of the quality of organic matter as food potentially available to consumers Danovaro and Fabiano 1997. The peak of BPC: TOC ratio in January 1997 was related to the high protein concentration at that time, pointing out the high quality of the organic matter. These results were reflected by the low $\mathrm{C}$ : $\mathrm{N}$ ratio values (maximum of 8) found for this month, indicative of a highly nutritive organic matter (Parsons, 1975) consisting mainly of relative easily biodegradable material. Similar results were found by Eabiano et_al_(1995) for the Ligurian Sea (NW Mediterranean). These findings coupled with the significant inverse relationship between $\mathrm{C}: \mathrm{N}$ ratio and $\mathrm{BPC}$ : TOC ratio $(P<0.001)$ suggest that $\mathrm{C}: \mathrm{N}$ ratio is related to the quality of the sedimentary organic matter in Barraña, especially when BPC: TOC values are under the threshold of $30 \%$ (Fig. 4b).

In agreement with Eabiano et_al_(1995) the temporal pattern of OM composition in surface sediments showed an inverse relationship between amounts of organic matter and its potential availability to consumers: low quantities ( TOC $=2.1 \mathrm{mg} \mathrm{g}^{-1}$ ) of high-quality organic matter in January 1997 (BPC: TOC ratio $=94 \%, 5-10 \mathrm{~cm}$ ) were replaced by large quantities (TOC $=7.7 \mathrm{mg} \mathrm{g}^{-1}$ ) of refractory material in July 1997 (BPC: TOC ratio $=14 \%, 20-25 \mathrm{~cm}$ ) at the medium tidal level. Sedimentary $\mathrm{OM}$ in summer was therefore mostly composed of refractory material that was largely unavailable to consumers. Correlation between TOC and $\mathrm{C}$ : $\mathrm{N}$ ratio $(\mathrm{R}=0.75, \mathrm{~N}=75, P<0.01)$ lead to a similar conclusion, indicating that high amounts of organic carbon were related to an increase in the refractory fraction.

Also the analysis of the PRT: CHO ratio supported these conclusions. Since proteins are more readily utilised by bacteria than carbohydrates Newell and Field, 1983) and are rapidly bound into refractory compounds, low values of
PRT: CHO ratio suggest the presence of aged OM Danovaro et al. 1993 and a role of labile proteins as a potentially limiting factor for benthic consumers Uumars and Wheat croft, 1989: Fabiano et al. 1995) PRT: CHO ratio reached 17.3 in January 1997 at the medium tidal level indicating the presence of newly-produced matter. Conversely, for the rest of the study period, PRT: CHO ratio remained below 3.5, confirming that the sediments of the intertidal flat were characterised, most part of the year, by a large amount of aged and/or non-living organic matter. The latter values (PRT: $\mathrm{CHO}<3.5$ ) are comparable to those observed in the Arno Estuary (PRT: $\mathrm{CHO}=0.3$ to 3.6; Eabiano and Danovaro. 1994) and higher than those reported from the Ligurian Sea (PRT: $\mathrm{CHO}=0.14$; Eabiane et al. 1995) and from Eastern Mediterranean Sea (PRT: $\mathrm{CHO}=0.09$; Danovaro et al. 1993. However, these comparisons must be viewed with caution since different methods for carbohydrate determination have been applied. Moreover, such high PRT: CHO values found in Barraña could have been overestimated as Dubois et al. (1956) method has been used for carbohydrate determination.

Although BPC and TOC concentrations at the high tidal level were lower than those found at the low tidal level, the BPC: TOC ratio was higher at the former. In contrast to what happened at the medium tidal level, these results indicate that high BPC: TOC values are not necessarily associated with large amounts of lipids, proteins and carbohydrates. Thus, though the concentration of biochemical compounds was higher at the low tidal level, food quality was better at the high tidal level (especially in April and October 1997). This suggests an increase in the proportion of labile material quality towards the high tidal level of the beach.

\section{Conclusions}

Results from the present study revealed significant temporal and spatial (i.e. among tidal levels) changes in the quantity and quality of the sedimentary OM, as well as significant variations between sediment layers. Differences on OM quantity between medium tidal level and high and low tidal levels appear connected to the presence of large amounts of OM. Particularly high protein concentrations in January and April 1997 at the medium tidal level suggest that Barraña intertidal flat might be an environment where 'natural' seasonal variation in biochemical composition of the sedimentary OM could be masked by episodic allochthonous inputs. Low BPC: TOC ratios observed for most part of the study period at the medium tidal level indicate that large part of $\mathrm{OM}$ is unaccounted for by labile compounds. Such high amounts of sedimentary OM were therefore of low nutritional value and not directly available to benthic consumers as such tended to be buried and accumulated into deeper sediment layers. 


\section{Acknowledgements}

This research was supported by the Xunta de Galicia (Xuga 30105A98) and the Universidade de Vigo (64102C859). The authors would like to thank "Equipo de Bentos" for their useful help during sampling. Special thanks to Alberto Velando and Ibone Amezaga for precious suggestions and criticisms on improving the manuscript. Thanks are also due to anonymous referees for suggestions leading to improvement of the manuscript.

\section{References}

Adam, P., 1990. Saltmarsh Ecology. Cambridge University Press, Cambridge.

Bligh, E.G., Dyer, W., 1959. A rapid method for total lipid extraction and purification. Can. J. Biochem. Physiol. 37, 911-917.

Bock, M.J., Miller, D.C., 1995. Storm effects on particulate food resources on an intertidal sandflat. J. Exp. Mar. Biol. Ecol. 187, 81-101.

Bretschko, G., Leichtfried, M., 1987. The determination of organic matter in river sediments. Arch. Hydrobiol (Suppl. 68), 403-417.

Buchanan, J.B., Longbottom, M.R., 1970. The determination of organic matter in marine muds: the effect of the presence of coal and the routine determination of proteins. J. Exp. Mar. Biol. Ecol. 5, 158-169.

Buscail, R., Pocklington, R., Germain, C., 1995. Seasonal variability of the organic matter in a sedimentary coastal environment: sources, degradation and accumulation (continental shelf of the Gulf of Lionsnorthwestern Mediterranean Sea). Cont. Shelf Res. 15, 843-869.

Cough, M.A., Mantoura, R.F.C., 1990. Advanced analytical methods for the characterization of macromolecular marine organic matter. In: Hilfe, E.R., Tuszguski, W (Eds.), Proc. Particle Desorption Mass Spectrometry for Marine Organic Chemistry, Third International Workshop on the Physics of Small Systems. World Scientific Press, Singapore, pp. 114-130.

Danovaro, R., 1996. Detritus-bacteria-meiofauna interactions in a seagrass bed (Posidonia oceanica) of the NW Mediterranean. Mar. Biol. 127, $1-13$.

Danovaro, R., Fabiano, M., 1995. Seasonal and interannual variation of benthic bacteria in a seagrass bed of the Mediterranean Sea: relationship with labile organic compounds and other environmental factors. Aquat. Microb. Ecol. 9, 17-26.

Danovaro, R., Fabiano, M., 1997. Seasonal changes in quality and quantity of food available for benthic suspension-feeders in the Golfo Marconi (North-Western Mediterranean). Estuarine, Coastal and Shelf Sci. 44, 726-733.

Danovaro, R., Fabiano, M., Della, Croce, N., 1993. Labile organic matter and microbial biomasses in deep-sea sediments (Eastern Mediterranean Sea). Deep-Sea Res. 40, 953-965.

Danovaro, R., Fabiano, M., Boyer, M., 1994. Seasonal changes of benthic bacteria in a seagrass bed (Posidonia oceanica) of the Ligurian Sea in relation to origin, composition and fate of the sediment organic matter. Mar. Biol. 119, 489-500.

Danovaro, R., Fraschetti, S., Belgrano, A., 1995. The potential impact of meiofauna on the recruitment of macrobenthos in a subtidal coastal benthic community of the Ligurian Sea (northwestern Mediterranean): a field study. In: Olsen, Olsen (Eds.), Proceedings of the XXVIII European Marine Biology Symposium, Fredesborg, Denmark. pp. 115-122.

Dell'Anno, A., Fabiano, M., Mei, M.L., Danovaro, R., 2000. Enzymatically hydrolysed protein and carbohydrate pools in deep-sea sediments: estimates of the bioavailable fraction and methodological considerations. Mar. Ecol. Prog, Ser. 196, 15-23.
Dubois, M., Gilles, K.A., Hamilton, S.K., Rebers, P.A., 1956. Colorimetric method for determination of sugars and related substances. Anal. Chem. 28, 350-356.

Duineveld, G.C.A., Lavaleye, M.S.S., Berghuis, E.M., Wilde, P.A.W.J., Weele, J., Kok, A., Batten, S.D., Leeuw, J.W., 1997. Patterns of benthic fauna and benthic respiration on the Celtic continental margin in relation to the distribution of phytodetritus. Int. Rev. Ges. Hydrobiol. 83, 395-424.

Emery, K.O., 1961. A sample method of measuring beach profiles. Limnol. Oceanogr. 6, 90-93.

Fabiano, M., Danovaro, R., 1994. Composition of organic matter in sediments facing a river estuary (Tyrrhenian Sea): relationships with bacteria and microphytobenthic biomass. Hydrobiol. 277, 71-84.

Fabiano, M., Danovaro, R., Fraschetti, S., 1995. A three-year time series of elemental and biochemical composition of organic matter in subtidal sandy sediments of the Ligurian Sea (northwestern Mediterranean). Cont. Shelf Res. 15, 1453-1469.

Fabiano, M., Chiantore, M., Povero, P., 1997. Short-term variations in particulate matter flux in Terra Nova Bay, Ross Sea. Antartic Sci. 9, 143-149.

Fichez, R., 1991. Composition and fate of organic matter in submarine cave sediments; implications for the biogeochemical cycle of organic carbon. Oceanol. Acta 14, 369-377.

Fichez, R., Dennis, P., Fontaine, M.F., 1993. Isotopic and biochemical composition of particulate organic matter in a shallow water estuary (Great Ouse, North Sea, England). Mar. Chem. 43, 263-276.

Gerchacov, S.M., Hatcher, P.G., 1972. Improved technique for analysis of carbohydrates in the sediment. Limnol. Oceanogr. 17, 938-943.

Graf, G., 1989. Pelagic-benthic coupling in a deep-sea benthic community. Nature 341, 437-439.

Grant, J., Hargrave, B.T., 1987. Benthic metabolism and the quality of sediment organic carbon. Biol. Ocean. 4, 243-264.

Grant, J., Bathmann, U.V., Mills, E.L., 1986. The interaction between benthic diatom films and sediment transport. Estuar. Coastal Shelf Sci. 23, 225-238.

Guitián, F., Carballas, J., 1976. Técnicas de análisis de suelos. In: Pico Sacro (Ed.), Santiago de Compostela. pp. 288-288.

Hargrave, B.T., Phillips, G.A., Doucette, L.I., 1995. Biochemical observations to assess benthic impacts of organic enrichment from marine aquaculture in the Western isles region of the Bay of Fundy, 1994. Can. Tech. Rep. Fish. Aquat. Sci. 2068.

Ittekkot, V., 1988. Global trends in the nature of organic matter in river suspensions. Nature 332, 436-438.

Josefson, A.B., Conley, D.J., 1997. Benthic response to a pelagic front. Mar. Ecol. Prog. Ser. 147, 49-62.

Jumars, P.A., Wheatcroft, R.A., 1989. Response of benthos to changing food quality and quantity, with a focus on deposit-feeding and bioturbation. In: Berger, W.H., Smetacek, V.S., Wefer, G (Eds.), Productivity of the oceans and past. Wiley \& Sons, S. Bernhard, Dahlem Konferenzen, pp. 235-253.

Lowry, O.H., Rosebrough, N.J., 1951. Protein measurement with the folin phenol reagent. J. Biol. Chem. 193, 265-275.

Manini, E., Danovaro, R., Fabiano, M., 1999. Early diagenesis of sedimentary organic matter and bacterial response in a frontal system of the Adriatic Sea: preliminary results. Atti XIII Congr. AIOL 13, 271-279.

Markwell, M.A.K., Haas, S.M., Bieber, L.L., Tolbert, M.E., 1978. A modification of the Lowry procedure to simplify protein determination in membrane and lipoprotein samples. Ann. Biochem. 87, 206-210.

Marsh, J.B., Weinstein, W.J., 1966. A simple charring method for determination of lipids. J. Lip. Res. 7, 574-576.

Mayer, L.M., 1989. The nature and determination of non-living sedimentary organic matter as a food source for deposit feeders. In: López, G., Tagon, G., Levinton, J. (Eds.), Ecology of marine deposit-feeders, Lecture Notes on Coastal and Estuarine Studies. Springer-Verlag, New York, pp. 98-113. 
Metzler, G.M., Smock, L.A., 1990. Storage and dynamics of subsurface detritus in a sand-bottomed stream. Can. J. Fish. Aquat. Sci. 47, 588-594.

Meyer-Reil, L.A., 1983. Benthic response to sedimentation events during autumn to spring at a shallow water station in the Western Kiel Bight. II. Analysis of benthic bacterial populations. Mar. Biol. 77, 247-256.

Meziane, T., Bodineau, L., Retiere, C., Thoumelin, G., 1997. The use of lipid markers to define sources of organic matter in sediment and food web of the intertidal salt-marsh-flat ecosystem of Mont-Saint-Michel Bay, France. J. Sea Res. 38, 47-58.

Newell, R.C., Field, J.G., 1983. The contribution of bacteria and detritus to carbon and nitrogen flow in a benthic community. Mar. Biol. Letters 4, 23-36.

Nie, N.H., Hull, J.G., Jenkins, G.J., Steinbrenner, K., Bent, D.H., 1975. SPSS-Statistical package for the social sciences, 2 edition. McGrawHill, New York, pp. 675-675.

Nordstrom, K.F., 1992. Estuarine beaches. Elsevier Applied Science, London and New York.

Parker, J.C., 1983. A comparison of methods used for the measurement of organic matter in marine sediments. Chem. Ecol. 1, 201-210.

Parsons, T.R., 1975. Particulate organic carbon in the sea. In: Riley, J.P., Skirrow, G (Eds.), Chemical Oceanography, 2nd edition. Academic Press, London.
Rowe, G.T., Deming, J.W., 1985. The role of bacteria in the turnover of organic carbon in deep-sea sediments. J. Mar. Res. 43, 925-950.

Saliot, A., 1984. Biogéochimie de la matière organique en milieu estuarien: stratégies d'échantillonnage et de recherche élaborées en Loire. France. Oceanol. Acta 7, 191-207.

Sargent, J.R., Hopkins, C.C.E., Seiring, J.V., Youngson, A., 1983. Partial characterization of organic material in surface sediments from Balsfiorden, northern Norway, in relation to its origin and nutritional value for sediment-ingesting animals. Mar. Biol. 76, 87-94.

Sokal, R.R., Rohlf, F.J., 1995. Biometry. WH Freeman \& Company, New York.

Spitzy, A., Ittekkot, V., 1991. Dissolved and particulate organic matters in rivers. In: Mantoura, R.F.C., Martin, J.M., Wollast, R (Eds.), Ocean Margin Processes in Global Change. Wiley \& Sons, Chichester, pp. 5-17.

Tenore, K.R., Hanson, R.B., 1980. Availability of detritus of different types and ages to a polychaete macroconsumer Capitella capitata.. Limnol. Oceanogr. 25, 553-558.

Zavattarelli, M., 1986. Moto ondoso e materiale detritico sospeso. PhD thesis, University of Genova. 